\title{
Molecular characteristics of pediatric non-ependymal, non-pilocytic gliomas associated with resistance to temozolomide
}

\author{
TAKETO EZAKI $^{1}$, HIKARU SASAKI ${ }^{1}$, YUICHI HIROSE ${ }^{2}$, TOMORU MIWA ${ }^{1}$, \\ KAZUNARI YOSHIDA ${ }^{1}$ and TAKESHI KAWASE ${ }^{1}$ \\ ${ }^{1}$ Department of Neurosurgery, Keio University School of Medicine, Tokyo 160-8582; \\ ${ }^{2}$ Department of Neurosurgery, Fujita Health University School of Medicine, Aichi 470-1192, Japan
}

Received April 7, 2011; Accepted August 2, 2011

DOI: $10.3892 / \mathrm{mmr} .2011 .573$

\begin{abstract}
Temozolomide constitutes current standard of care for adult patients with high-grade gliomas. However, results for pediatric gliomas are rather disappointing. In order to investigate the molecular differences between pediatric and adult gliomas that could affect sensitivity to temozolomide, we studied 23 pediatric non-ependymal, non-pilocytic gliomas in comparison to 59 consecutive adult gliomas for the expression of $\mathrm{O}^{6}$-methylguanine methyltransferase (MGMT) and the DNA mismatch repair protein, mutS homolog 6 (MSH6) by immunohistochemistry, as well as for the presence or absence of promoter methylation of the MGMT gene by methylationspecific PCR. The expression of MGMT in pediatric gliomas was significantly higher than in adult gliomas, as shown by immunohistochemistry $(\mathrm{p}=0.00004)$. This association was conserved if statistical analysis was carried out only in astrocytic tumors (diffuse astrocytoma, anaplastic astrocytoma and glioblastoma, $\mathrm{p}=0.00007$ ), or in oligodendroglial tumors (oligodendroglioma and anaplastic oligodendroglioma, $\mathrm{p}=0.020$ ). Although methylation-specific PCR was successfully performed only in 15 pediatric gliomas, it also showed a trend toward less frequent methylation in pediatric as opposed to adult gliomas $(\mathrm{p}=0.242)$. MSH6 was almost equally expressed in pediatric and adult gliomas. Pediatric gliomas appear to have a distinct molecular profile associated with resistance to temozolomide. Higher expression of MGMT and a trend toward less frequent methylation of the promoter region of $M G M T$ gene may partly account for relative resistance to temozolomide in pediatric gliomas as compared to adult gliomas.
\end{abstract}

Correspondence to: Dr Hikaru Sasaki, Department of Neurosurgery, Keio University School of Medicine, 35 Shinanomachi, Shinjyuku-ku, Tokyo 160-8582, Japan

E-mail: sasaki@sc.itc.keio.ac.jp

Key words: pediatric gliomas, temozolomide resistance, $\mathrm{O}^{6}$-methylguanine methyltransferase, mutS homolog 6 , methylation-specific PCR

\section{Introduction}

Temozolomide, an oral methylating agent, has better activity and less toxicity than conventional agents, including nitrosoureas in the treatment of gliomas $(1,2)$. Indeed, concomitant and adjuvant temozolomide has improved progression-free survival and overall survival compared to radiotherapy alone in adult patients with newly diagnosed supratentorial glioblastoma, and constitutes current standard of care for those patients $(3,4)$. Nowadays, the majority of clinical trials for gliomas comprises protocols, including temozolomide. However, the efficacy of temozolomide is virtually based on reports on adult gliomas, and results for pediatric gliomas are rather disappointing. Verschuur et al treated 20 children with recurrent high-grade gliomas, including 11 grade III and 8 grade IV gliomas. Progression-free survival at 6 months was 18 and $12.5 \%$ for each grade (5). Those figures are less favorably compared to those reported in adult studies $(1,2)$. Ruggiero et al and Lashford et al also reported lower response rate to temozolomide in recurrent pediatric, high-grade gliomas as compared to adult gliomas $(6,7)$. Moreover, in a previous phase II trial (ACNS0126) for newly diagnosed pediatric high-grade gliomas, chemoradiotherapy with temozolomide only provided comparable results to the historical control with BCNU (8).

Temozolomide rapidly hydrolyzed into 5 -amino- $1 \mathrm{H}$ - imidazole-4-carboxamide and methyl diazonium ion at physiological $\mathrm{pH}$ via 5-[(1z)-3-Methyl-1-en-1-yl]-1H-imidazole-4-carboxamide. The cytotoxity of temozolomide is caused by methyl diazonium ion $(9,10)$. The methylating activity of the methyl diazonium ion is exerted on the $\mathrm{N}^{7}$-position (70\%), $\mathrm{N}^{3}$-position $(10 \%)$ and $\mathrm{O}^{6}$-position of guanine (9\%) (11). However, methylation of the $\mathrm{N}^{7}$-guanine is, in most cases, repaired by the base excision repair system in cancer cells. However, methylation of the $\mathrm{O}^{6}$-guanine leads to a mispaired thymine (GT-mismatch) in DNA reproduction (12), and finally leads to DNA strand break through the futile mismatch repair cycle (13). Therefore, a defect in the mismatch repair system as well as the increased expression of $\mathrm{O}^{6}$-methylguanine methyltransferase (MGMT), the enzyme that removes alkyl group from $\mathrm{O}^{6}$-guanine, appear to be associated with resistance to temozolomide in gliomas. Indeed, the expression of the mismatch repair protein, mutS homolog 6 (MSH6), is lost in $41 \%$ of recurrent glioblastomas in comparison to $0 \%$ of pre-treatment glioblastomas, and loss 
of MSH6 may be associated with progression during temozolomide treatment (14).

Recent studies suggest that pediatric gliomas have unique genetic characteristic and/or expression profiles associated with particular signaling pathways as compared to adult tumors (15). We therefore examined molecular factors that could affect sensitivity to temozolomide in pediatric gliomas in comparison to adult gliomas. We studied the expression of MGMT and the expression of the DNA mismatch repair protein, MSH6, by immunostaining, as well as the presence or absence of promoter methylation of the MGMT gene by methylation-specific PCR.

\section{Materials and methods}

Tumor samples. Formalin-fixed, paraffin-embedded tissues of non-ependymal, non-pilocytic gliomas from 23 pediatric ( $\leq 20$ years of age) patients were collected from the tissue bank at the Department of Neurosurgery, Keio University, Tokyo, Japan. All were intracranial tumors and histologically proven. For comparison, 59 consecutive cases of adult glioma newly diagnosed between 2005 and 2009 were also analyzed. Pathological classifications were made according to the World Health Organization (WHO) criteria (16). No cases had any prior adjuvant therapy (radiation or chemotherapy).

Immunohistochemistry of MGMT and MSH6. Immunohistochemistry was performed on the formalin-fixed, paraffin-embedded sections. Microwave antigen retrieval was carried out in $10 \mathrm{mM}$ sodium citrate buffer of pH 6.0 (MGMT) or of $\mathrm{pH} 7.0$ (MSH6) (14,17). Sections were incubated with mouse monoclonal antibody against MGMT (clone MT 3.1; Dako, Glostrup, Denmark) or MSH6 (clone 44; BD Biosciences, San Jose, CA, USA). Vascular endothelial cells and brain metastasis of colon adenocarcinoma were used as the positive control for MGMT and MSH6, respectively. The staining indices were analyzed in a total of $>1,000$ tumor cells on more than three areas showing typical appearance of each tumor.

Methylation-specific PCR for MGMT gene. Tumor DNA was extracted from a microdissected piece of the formalin-fixed, paraffin-embedded sections. Tissue microdissection was performed referring to the pathological findings of consecutive sections that were stained with $\mathrm{H} \& \mathrm{E}$ or mouse monoclonal anti Ki-67 antibody (MIB-1; Dako) to select areas showing typical appearance of each tumor. Bisulfate reaction and subsequent PCR were performed using the EZ DNA Methylation-Direct ${ }^{\mathrm{TM}}$ kit (Zymo Research Corp., Orange, CA, USA). PCR was performed with primers specific for methylated or unmethylated DNAs (Sigma-Aldrich Japan, Inc.) and Taq polymerase HotStart Version (Takara Taq ${ }^{\text {TM }}$ HotStart Version; Takara Bio, Inc., Shiga, Japan), and the annealing temperature was decreased from 65 to $58^{\circ} \mathrm{C}$ with 35 cycles in total (18-23). The PCR products were separated on a $4 \%$ MetaPhor Agarose gel (Lonza, Rockland, ME, USA). Control DNAs for the methylated, unmethyled and wild-type sequence were obtained in the Chemicon CpG WIZ MGMT Amplification kit (Temecula, CA, USA).

Statistical analysis. The F-test and Student's t-test were used to compare staining indices of MGMT or MSH6, and $\chi^{2}$ test was
Table I. Patient characteristics.

\begin{tabular}{lc}
\hline $\begin{array}{l}\text { Variable } \\
\text { (histology) }\end{array}$ & $\begin{array}{c}\text { No. of patients } \\
\text { (pediatric/adult) }\end{array}$ \\
\hline Glioblastoma & $3 / 24$ \\
Anaplastic astrocytoma & $6 / 7$ \\
Diffuse astrocytoma & $11 / 7$ \\
Anaplastic oligodendroglioma & $1 / 7$ \\
Oligodendroglioma & $2 / 12$ \\
Oligoastrocytoma & $0 / 2$ \\
Age (years) & \\
S20 & 23 \\
$>20$ & 59 \\
Gender & \\
Male & $13 / 35$ \\
Female & $10 / 24$ \\
\hline
\end{tabular}

used to compare frequency of the methylation of the MGMT promoter between pediatric and adult astrocytic gliomas.

\section{Results}

Patient characteristics. Clinical data of 23 patients with pediatric non-ependymal, non-pilocytic gliomas in comparison to 59 patients with newly diagnosed adult gliomas are given in Table I. There were 13 males and 10 females with ages ranging from 1 to 20 years (median, 10). The pediatric tumors were classified as diffuse astrocytoma in 11, anaplastic astrocytoma in 6, glioblastoma in 3, oligodendroglioma in 2 and anaplastic oligodendroglioma in 1 patient. Ten tumors were located in the cerebrum, 9 in the brain stem, 2 in the thalamus and 2 in other locations (1 optic nerve and 1 pineal lesion).

Immunohistochemical analysis of MGMT and MSH6. Immunohistochemistry of MGMT and MSH6 was performed on 21 and 12 (due to lack of tissues) pediatric gliomas for each, and the results were compared to those of adult gliomas (Table II). For both MGMT and MSH6, positive staining was found in the nuclei and representative slides are shown in Fig. 1. The expression of MGMT was significantly higher in the pediatric gliomas than in the adult gliomas $(\mathrm{p}=0.00004)$. This association was also found if the statistical analysis was limited to include only astrocytic gliomas (diffuse astrocytoma, anaplastic astrocytoma and glioblastoma, $\mathrm{p}=0.00007$ ) or to include only astrocytic gliomas of grade 2 and 3 (diffuse astrocytoma and anaplastic astrocytoma, $\mathrm{p}=0.0001$ ) in order to eliminate the influence of difference in the number of glioblastomas between the pediatric and adult groups. Moreover, a difference in the expression of MGMT was also significantly noted in oligodendroglial tumors $(p=0.020)$. MSH6 was almost equally expressed in pediatric and adult gliomas, and glioblastomas showed a trend toward higher expression than the other lower grade gliomas in both the pediatric $(\mathrm{p}=0.520)$ and adult ( $\mathrm{p}=0.017)$ groups (Table II).

Methylation-specific PCR. Methylation-specific PCR of the promoter region of the MGMT gene was successfully 
Table II. Immunohistochemical analysis for MGMT and MSH6.

\begin{tabular}{lcc}
\hline Histology & $\begin{array}{c}\text { MGMT average (\%) } \\
\text { (no. of pediatric/ } \\
\text { no. of adult) } \\
\text { tumors }\end{array}$ & $\begin{array}{c}\text { MSH6 average }(\%) \\
\text { (no. of pediatric/ } \\
\text { no. of adult) } \\
\text { tumors }\end{array}$ \\
\hline GM & $41.2(11.0)$ & $49.7(35.7)$ \\
AA & $3 / 24$ & $2 / 14$ \\
& $39.2(6.47)$ & $0.7(7.4)$ \\
DA & $5 / 7$ & $4 / 2$ \\
& $64.2(20.0)$ & $5.22(8.9)$ \\
AO & $10 / 7$ & $5 / 3$ \\
& $97.8(4.83)$ & $\mathrm{N} / \mathrm{M}(0)$ \\
OD & $1 / 7$ & $0 / 1$ \\
& $7.7(4.72)$ & $0(0)$ \\
OA & $2 / 12$ & $1 / 2$ \\
& $\mathrm{~N} / \mathrm{M}(7.7)$ & $\mathrm{N} / \mathrm{M}(15.7)$ \\
\hline
\end{tabular}

GM, glioblastoma; AA, anaplastic astrocytoma; DA, diffuse astrocytoma; AO, anaplastic oligodendroglioma; OD, oligodendroglioma; OA, oligoastrocytoma; N/M, no material.
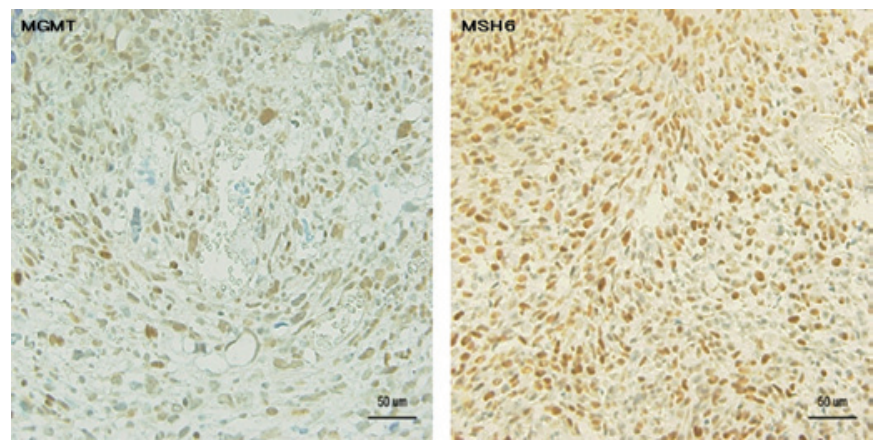

Figure 1. Representative immunostaining for MGMT and MSH6. Left, MGMT immunohistochemistry in pediatric glioblastoma. Right, MSH6 immunohistochemistry in pediatric glioblastoma. For both, positive staining was found in the nuclei.

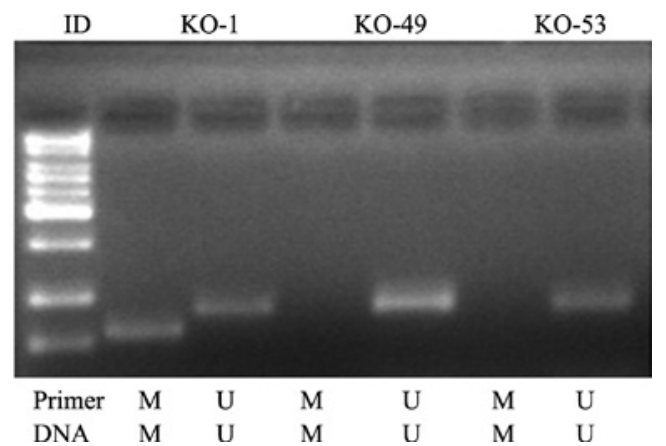

Figure 2. Methylation-specific PCR of the promoter region of the MGMT gene. KO-1, methylated; KO-49, unmethylated; KO-53, unmethylated.

performed in 15 pediatric gliomas (Fig. 2), and showed a trend toward less frequent methylation in the pediatric gliomas as opposed to the adult gliomas ( $p=0.242$; Figs. 3 and 4$)$. This

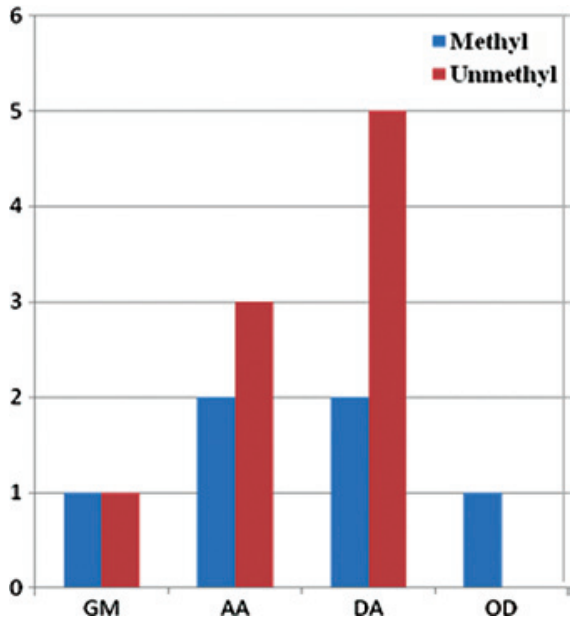

Figure 3. Results of methylation-specific PCR for the $M G M T$ promoter in pediatric gliomas $[\mathrm{n}=15$; glioblastoma $(\mathrm{GM})=2$, anaplastic astrocytoma $(\mathrm{AA})=5$, diffuse astrocytoma $(\mathrm{DA})=7$ and oligodendroglioma $(\mathrm{OD})=1$.

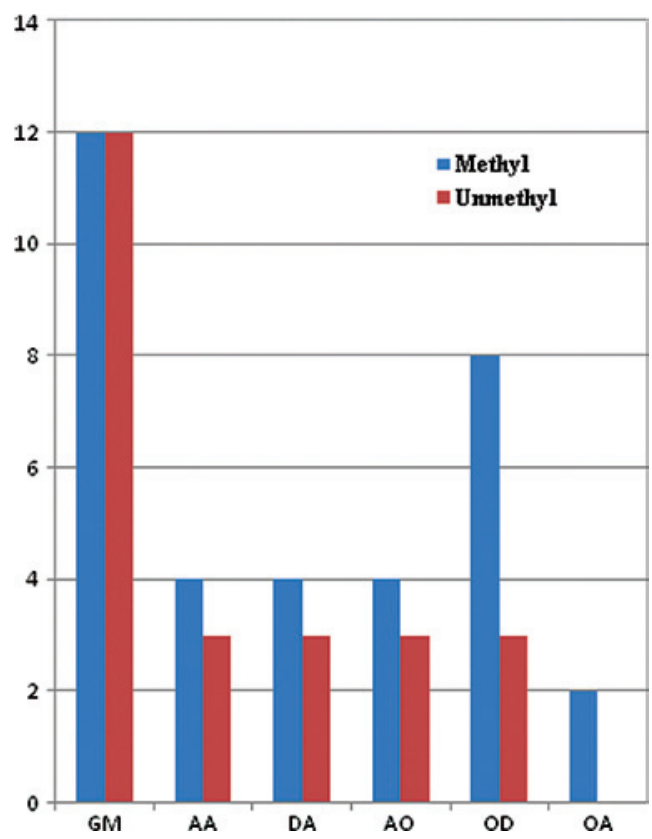

Figure 4. Results of methylation-specific PCR for the MGMT promoter in adult gliomas $[\mathrm{n}=59$; glioblastoma $(\mathrm{GM})=24$, anaplastic astrocytoma $(\mathrm{AA})=7$, diffuse astrocytoma $(\mathrm{DA})=7$, anaplastic oligodendroglioma $(\mathrm{AO})=7$, oligoden droglioma $(\mathrm{OD})=12$ and oligoastrocytoma $(\mathrm{OA})=2$ ]

association was also found if statistical analysis was carried out only in astrocytic gliomas (diffuse astrocytoma, anaplastic astrocytoma and glioblastoma, $\mathrm{p}=0.378$ ), and in astrocytic gliomas of grade 2 and 3 (diffuse astrocytoma and anaplastic astrocytoma, $\mathrm{p}=0.299$ ) where the influence of difference in the number of glioblastomas was excluded. No difference in the MGMT methylation status in oligodendroglial tumors between the pediatric and adult groups was detected, possibly due to the small number of cases analyzed in the pediatric group.

\section{Discussion}

The effectiveness of temozolomide, alone or combination with radiation and/or other agents, in pediatric gliomas remains 
uncertain (24). Regardless of the number of patients, to date, there have been no reports showing clinical results supporting the efficacy of temozolomide. Although studies on MGMT in pediatric gliomas are sparse, there have been some recent studies. Pollack et al detected the overexpression (more than $25 \%$ positivity) of MGMT in 25 out of $68(37 \%)$ pediatric high-grade gliomas by immunohistochemistry (26). Schlosser et al studied the methylation status of the MGMT promoter in 24 pediatric high-grade gliomas by the quantitative method, and found that MGMT methylation was associated with an elevated event-free survival only if methylation was over $14 \%$ (27). However, none of these studies compared the MGMT status between pediatric and adult gliomas. To our knowledge, this is the first study showing that the expression of MGMT is significantly higher in pediatric astrocytic tumors as well as oligodendroglial tumors than in their adult counterparts. Although methylation-specific PCR was successfully performed only in 15 pediatric gliomas, our study also shows a trend toward less frequent methylation in pediatric, as opposed to adult gliomas. The number of cases was small, however, the present findings may partly explain the inferior outcome of temozolomide therapy in pediatric gliomas, as opposed to adult ones.

The difference in MGMT expression between pediatric and adult gliomas could be due to the fact that pediatric gliomas are associated with distinct molecular characteristics compared to adult tumors. Indeed, in the present series of pediatric gliomas, a single copy gain of chromosome $1 \mathrm{q}$ was frequently found (50\% in 20 tumors) as opposed to adult gliomas (2.5\% in 170 tumors) (15). Moreover, codeletion of $1 \mathrm{p} / 19 \mathrm{q}$, the genetic hallmark of oligodendrogliomas in adults, was not observed in the 3 oligodendroglial tumors included in the present study (15).

Loss of expression and mutation of the mismatch repair gene, MSH6, may be associated with glioblastoma progression during temozolomide treatment $(14,25)$. In the present study, however, there was no difference in MSH6 immunohistochemistry between pediatric and adult gliomas. Pollack et al found microsatellite instability only in 3 of 68 pediatric malignant gliomas, and suggested that mismatch repair deficiency is an uncommon mechanism of alkylator resistance in those tumors (26). Although the number of cases was small, our study, similar to the one by Pollack et al, does not show any evidence suggesting the association of MSH6 status with the difference in sensitivity to temozolomide between pediatric and adult gliomas.

The present results suggest that the higher expression of MGMT may be associated with relative resistance to temozolomide in pediatric gliomas as compared to adult gliomas. Therefore, to improve the effectiveness of temozolomide chemotherapy in pediatric gliomas, the depletion of MGMT with guanine analogues (e.g., $\mathrm{O}^{6}$-benzylguanine and interferon $\beta)$ is a theoretically reasonable treatment strategy $(28,29)$. Inhibition of the self repair of $\mathrm{N}^{7}$-methylguanine with PARP inhibitor (e.g., BSI-201) may also be practical as a different approach (30). Further elucidation of the molecular characteristics of pediatric gliomas is important for the effective treatment and better outcome of the patients with those tumors.

In conclusion, non-ependymal, non-pilocytic, pediatric gliomas are associated with higher expression of MGMT, and with a trend toward the less frequent methylation of the MGMT promoter, as opposed to adult gliomas. However, MSH6 was equally expressed in pediatric and adult gliomas. Although the number of cases was small, these results suggest that the difference in MGMT status partly accounts for the different sensitivity to temozolomide in pediatric gliomas as compared to adult gliomas. Moreover, together with our previous publication (15), these data clearly suggest that pediatric gliomas have distinct genetic characteristics from their adult counterparts.

\section{Acknowledgements}

The authors thank Ms. Kiyomi Koide and Ms. Naoko Tsuzaki for their technical assistance.

\section{References}

1. Yung WK, Prados MD, Yaya Tur R, et al: Multicenter phase II trial of temozolomide in patients with anaplastic astrocytoma or anaplastic oligoastrocytoma at first relapse. J Clin Oncol 17: 2762-2771, 1999

2. Yung WK, Albright RE, Olson J, et al: A phase II study of temozolomide vs. procarbazine in patients with glioblastoma multiforme at first relapse. Br J Cancer 83: 588-593, 2000.

3. Stupp R, Manson WP, van den Bent MJ, et al: Radiotherapy plus concomitant and adjuvant temozolomide for glioblastoma. N Engl J Med 352: 987-996, 2005.

4. Gilbert MR, Friedman HS, Kuttesch JF, et al: A phase II study of temozolomide in patients with newly diagnosed supratentorial malignant glioma before radiation therapy. Neuro Oncol 4: 261-267, 2002.

5. Verschuur AC, Grill J, Lelouch-Tubiana A, Couanet D, Kalifa C and Vassal G: Temozolomide in pediatric high-grade glioma: a key for combination therapy. Br J Cancer 91: 425-429, 2004.

6. Ruggerio A, Cefalo G, Garre ML, et al: Phase II trial of temozolomide in children with recurrent high-grade glioma. J Neurooncol 77: 89-94, 2006.

7. Lashford LS, Thiesse P, Jouvet A, et al: Temozolomide in malignant gliomas of childhood: a United Kingdom Children's Cancer Study Group and French Society for Pediatric Oncology Intergroup Study. J Clin Oncol 20: 4684-4691, 2002.

8. Cohen K: The treatment of high-grade gliomas in pediatrics: where have we been? Where are we going? Neuro Oncol 10: S427, 2008

9. Newlands ES, Blackledge GR, Slack JA, et al: Phase I trial of temozolomide (CCRG 81045 M\&B 39831 : NSC 362856). Br J Cancer 65: 287-291, 1992.

10. Brada M, Judson I Beale P, et al: Phase I dose-escalation and pharmacokinetic study of temozolomide (SCH 52365) for refractory relapsing malignancies. Br J Cancer 81: 1022-1030, 1999.

11. Tentori L and Graziani G: Pharmacological strategies to increase the antitumor activity of methylating agents. Curr Med Chem 9: 1285-1301, 2002.

12. Catapano CV, Broggni M, Erba E, et al: In vitro and in vivo methazolastone-induced DNA damage and repair in L-1210 leukemia sensitive and resistant to chloethylnitrosoureas. Cancer Res 47: 4884-4889, 1987.

13. Sarkaria JN, Kitange GJ, Jams CD, et al: Mechanisms of chemoresistance to alkylating agents in malignant glioma. Clin Cancer Res 14: 2900-2908, 2008.

14. Cahill DP, Levine KK, Betnsky RA, et al: Loss of mismatch repair protein MSH6 in human glioblastomas is associated with tumor progression during temozolomide treatment. Clin Cancer Res 13: 2038-2045, 2007.

15. Miwa T, Hirose Y, Sasaki H, Ezaki T, Yoshida K and Kawase T: Single copy gain of chromosome 1q is a negative prognostic marker in pediatric non-ependymal, non-pilocytic gliomas. Neurosurgery 68: 206-212, 2011.

16. Louis DN, Ohgaki H, Wiestler OD and Cavenee WK (eds): WHO Classification of the Tumours of the Central Nervous System. International Agency for Research on Cancer. Lyon, France, 2007.

17. Hunter C, Smith R, Cahill DP, et al: A hypermutation phenotype and somatic MSH6 mutations in recurrent human malignant gliomas after alkylator chemotherapy. Cancer Res 66: 3987-3991, 2006. 
18. Esteller M, Garcia-Foncillas J, Andion E, et al: Inactivation of the DNA-repair gene MGMT and the clinical response of gliomas to alkylating agents. N Engl J Med 343: 1350-1354, 2000.

19. Tentori L, Orlando L, Lacal PM, et al: Inhibition of $\mathrm{O}^{6}$-alkylguanine DNA-alkyltransferase or poly (ADP-ribose) polymerase increases susceptibility of leukemic cells to apotosis by temozolomide. Mol Pharmacol 52: 249-258, 1997.

20. Nakamura M, Watanabe T, Yonekawa Y, Kleihues P and Ohgaki H: Promoter methylation of DNA repair gene MGMT in astrocytomas in frequently associated with $\mathrm{G}: \mathrm{C} \rightarrow \mathrm{A}: \mathrm{T}$ mutations of the TP53 tumors suppressor gene. Carcinogenesis 22: 1715-1719, 2001.

21. Herman JG, Graff JR, Myohanen S Nelkin BD and Baylin SB: Methylation-specific PCR: a novel PCR assay for methylation status of CpG island. Proc Nat Acad Sci USA 93: 9821-9826, 1996.

22. Harris LC, Potter PM, Tano K Shiota S, Mitra S and Brent TP: Characterization of the promoter region of the human $\mathrm{O}^{6}$-methylguanine-DNA methyltransferase gene. Nucleic Acids Res 19: 6163-6167, 1991.

23. Rydberg B, Spurr N and Karran P: cDNA cloning and chromosomal assignment of the human $\mathrm{O}^{6}$-methylguanine-DNA methyltransferase. J Biol Chem 265: 9563-9569, 1990.

24. Bartes U, Baruchel S, Carret AS, et al: The use and effectiveness of temozolomide in children with central nervous system tumours: a survey from the Canadian Paediatric Brain Tumour Consortium. Curr Oncol 18: e19-e24, 2011
25. Yip S, Miao J, Cahill DP, et al: MSH6 mutations arise in glioblastomas during temozolomide therapy and mediate temozolomide resistance. Clin Cancer Res 15: 4622-4629, 2009.

26. Pollack IF, Hamilton RL, Sobol RW, et al: Mismatch repair deficiency is an uncommon mechanism of alkylator resistance in pediatric malignant. Pediatr Blood Cancer 55: 1066-1071, 2010.

27. Schlosser S, Wagner S, Mühlisch J, et al: MGMT as a potential stratification marker in relapsed high-grade glioma of children: the HIT-GBM experience. Pediatr Blood Cancer 54: 228-237, 2010.

28. Quinn JA, Jiang SX, Reardon DA, et al: Phase II trial of temozolomide plus $\mathrm{O}^{6}$-benzylguanine in adults with recurrent, temozolomide-resistant malignant glioma. J Clin Oncol 27: 1262-1267, 2009

29. Natsume A, Ishii D, Wakabayashi T, et al: IFN-beta downregulates the expression of DNA repair gene MGMT and sensitizes resistant glioma cells to temozolomide. Cancer Res 65: 7573-7579, 2005

30. O'Shaughnessy J, Osborne C, Pippen J, et al: Efficacy of BSI-201, a poly (ADP-ribose) polymerase-1 (PARP1) inhibitor, in combination with gemcitabine/carboplatin $(\mathrm{G} / \mathrm{C})$ in patients with metastatic triple-negative breast cancer (TNBC): results of a randomized phase II trial. 2009 ASCO Annual meeting, 2009. 